\title{
A The Journal of Chemical Physics
}

\section{Gas-to-cluster effects in S 2p-excited SF6}

Roman Flesch, Ertugrul Serdaroglu, Xenia O. Brykalova, Elena I. Kan, Ekaterina S. Klyushina, Yuri S.

Krivosenko, Andrey A. Pavlychev, and Eckart Rühl

Citation: The Journal of Chemical Physics 138, 144302 (2013); doi: 10.1063/1.4798975

View online: http://dx.doi.org/10.1063/1.4798975

View Table of Contents: http://scitation.aip.org/content/aip/journal/jcp/138/14?ver=pdfcov

Published by the AIP Publishing

\section{Articles you may be interested in}

Photodissociation of $(\mathrm{SO} 2 \cdots \mathrm{XH})$ Van der Waals complexes and clusters $(\mathrm{XH}=\mathrm{C} 2 \mathrm{H} 2, \mathrm{C} 2 \mathrm{H} 4, \mathrm{C} 2 \mathrm{H} 6)$ excited at $32040-32090 \mathrm{~cm}-1$ with formation of $\mathrm{HSO} 2$ and $X$

J. Chem. Phys. 140, 054304 (2014); 10.1063/1.4863445

Photoabsorption and S 2p photoionization of the SF6 molecule: Resonances in the excitation energy range of 200-280 eV

J. Chem. Phys. 134, 174311 (2011); 10.1063/1.3583815

Photoionization of iodine atoms: Rydberg series which converge to the I + ( S 10 ) $\leftarrow$ I ( P 23 / 2 ) threshold J. Chem. Phys. 132, 244304 (2010); 10.1063/1.3447382

Vibrational branching ratios in the ( $\mathrm{b} 2 \mathrm{u}$ ) - 1 photoionization of C $6 \mathrm{~F} 6$

J. Chem. Phys. 131, 044311 (2009); 10.1063/1.3180817

Anion and cation-yield spectroscopy of core-excited SF 6

J. Chem. Phys. 122, 094312 (2005); 10.1063/1.1855314

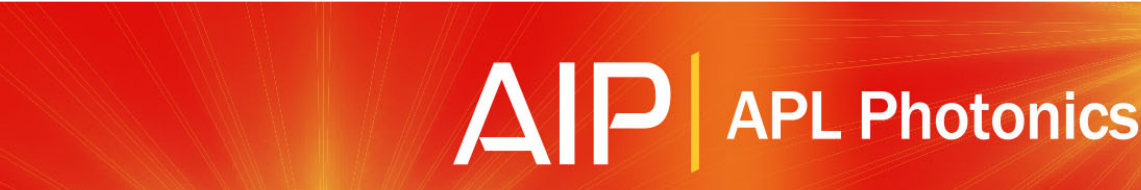 \\ APL Photonics is pleased to announce Benjamin Eggleton as its Editor-in-Chief}

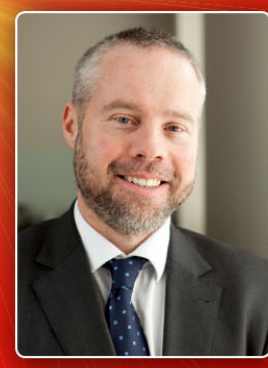




\title{
Gas-to-cluster effects in S $2 p$-excited $\mathrm{SF}_{\mathbf{6}}$
}

\author{
Roman Flesch, ${ }^{1}$ Ertugrul Serdaroglu, ${ }^{1}$ Xenia O. Brykalova, ${ }^{2}$ Elena I. Kan, ${ }^{2}$ \\ Ekaterina S. Klyushina, ${ }^{2}$ Yuri S. Krivosenko, ${ }^{2}$ Andrey A. Pavlychev, ${ }^{2}$ \\ and Eckart Rühl ${ }^{1}$ \\ ${ }^{1}$ Physikalische und Theoretische Chemie, Institut für Chemie und Biochemie, Freie Universität Berlin, \\ Takustr. 3, 14195 Berlin, Germany \\ ${ }^{2}$ Institute of Physics, St. Petersburg State University, St. Petersburg 198504, Russia
}

(Received 24 November 2012; accepted 19 March 2013; published online 10 April 2013)

\begin{abstract}
High resolution $\mathrm{X}$-ray spectroscopic studies on free $\mathrm{SF}_{6}$ molecules and $\mathrm{SF}_{6}$ clusters near the $\mathrm{S} 2 p$ ionization thresholds are reported. Spectral changes occurring in clusters for the intense molecular-like $\mathrm{S} 2 p_{1 / 2,3 / 2} \rightarrow 6 \mathrm{a}_{1 \mathrm{~g}^{-}}, 2 \mathrm{t}_{2 \mathrm{~g}^{-}}$, and $4 \mathrm{e}_{\mathrm{g}}$-resonances are examined in detail. Neither gas-to-cluster spectral shifts nor changes in peak shape are observed for the pre-edge $6 \mathrm{a}_{1 \mathrm{~g}}$-band. Significant changes in band shape and distinct gas-to-cluster shifts occur in the $\mathrm{S} 2 p_{1 / 2,3 / 2} \rightarrow 2 \mathrm{t}_{2 \mathrm{~g}}$ - and $4 \mathrm{e}_{\mathrm{g}}$-transitions. These are found in the $\mathrm{S} 2 p$-ionization continua. The quasiatomic approach is used to assign the experimental results. It is shown that a convolution of asymmetric and symmetric contributions from Lorentzian and Gaussian line shapes allows us to model the spectral distribution of oscillator strength for the $\mathrm{S} 2 p_{1 / 2,3 / 2} \rightarrow 2 \mathrm{t}_{2 \mathrm{~g}^{-}}$, and $4 \mathrm{e}_{\mathrm{g}}$-transitions. The asymmetry is due to trapping of the photoelectron within the finite size potential barrier. The Lorentzian contribution is found to be dominating in the line shape of the $\mathrm{S} 2 p \rightarrow 2 \mathrm{t}_{2 \mathrm{~g}}$ - and $4 \mathrm{e}_{\mathrm{g}}$-bands. The spectroscopic parameters of the spin-orbit components of both the $2 \mathrm{t}_{2 \mathrm{~g}}$ - and $4 \mathrm{e}_{\mathrm{g}}$-bands are extracted and their gas-to-cluster changes are analyzed. The photoelectron trapping times in free and clustered $\mathrm{SF}_{6}$ molecules are determined. Specifically, it is shown that spectral changes in clusters reflected in core-to-valence-transitions are due to a superposition of the singly scattered photoelectron waves at the neighboring molecules with the primary and multiply scattered waves within the molecular cage. (0) 2013 American Institute of Physics. [http://dx.doi.org/10.1063/1.4798975]
\end{abstract}

\section{INTRODUCTION}

During the last years, interest in research on Van-derWaals clusters has grown rapidly. ${ }^{1,2}$ Specifically, free variable size molecular Van-der-Waals clusters have been investigated in the soft X-ray regime. ${ }^{3-9}$ These are regarded as suitable model systems for studying size effects of elementary processes in weakly bound quantum systems as well as cage effects in composite and biological systems. In addition to significant changes in core-to-Rydberg transitions studied by high resolution X-ray spectroscopy, significantly smaller spectral shifts in core-to-valence transitions in molecular clusters have been observed. ${ }^{7,10}$ The latter ones indicate that the line shapes of the individual vibrational bands are almost unchanged. Small and distinct redshifts of $1 \mathrm{~s}$ $\rightarrow \pi^{*}$-transitions have been identified in $\mathrm{N}_{2}, \mathrm{CO}$, and benzene clusters including the gas-to-solid shift, which depends sensitively on the molecular system and are of the order of 2-60 meV. ${ }^{7-9}$ Even larger spectral shifts have been observed for aromatic molecules with a substantial dipole moment, whereby for $\mathrm{C} 1 s \rightarrow \pi^{*}$-excited pyridine a spectral redshift of $90 \mathrm{meV}$ is observed, whereas a spectral blueshift of the same magnitude is observed for the $\mathrm{N} 1 s \rightarrow \pi^{*}$-excitation. ${ }^{11}$ The gas-to-cluster redshifts have been on the one hand attributed to the freezing of molecular rotations and the dynamic stabilization of the core-excited clustered molecules. ${ }^{7}$ On the other hand, $a b$ initio calculations show for individual sites in clusters site-specific stabilization or destabi- lization effects, which explain the overall observed spectral shifts.

Clusters of $\mathrm{SF}_{6}$ are of specific interest, since they also show intense core-to-valence-transitions, which occur in the $\mathrm{S}$ $2 p$ core ionization continua. ${ }^{8,9}$ Striking similarities in spectral shape have been observed for the shape resonances in free and clustered $\mathrm{SF}_{6},{ }^{8,9}$ which is in full agreement with earlier low resolution spectroscopic work on the isolated molecule and the solid. ${ }^{12,13}$

$\mathrm{S} 2 p$-excited $\mathrm{SF}_{6}$ has been investigated before due to its unique cage structure and its double-well potential. ${ }^{14-16}$ The $2 \mathrm{t}_{2 \mathrm{~g}}$ - and $4 \mathrm{e}_{\mathrm{g}}$-shape resonances are the dominating features above the $\mathrm{S} 2 p$-ionization threshold. These resonances are known to be associated with temporary trapping of the photoelectron ejected from the sulfur core within the octahedral cage of electronegative fluorine atoms as well as with subsequent tunneling of the photoelectron through the cage into the S $2 p$-ionization continua. ${ }^{8,9,15,16}$ Another assignment links the resonances with electronic transitions from the deeply bound $2 \mathrm{t}_{1 \mathrm{u}}$-orbital to the unoccupied virtual $2 \mathrm{t}_{2 \mathrm{~g}}$ - and $4 \mathrm{e}_{\mathrm{g}}-$ orbitals, which are embedded in the $\mathrm{S} 2 p$-continua (see, e.g., Refs. 8, 9, 16, and 17). Both assignments can be considered to be equivalent.

Specifically, the $S 2 p \rightarrow 2 t_{2 g}$-shape resonance shows in clusters a spectral redshift of $35 \pm 5 \mathrm{meV}$, as compared to the isolated molecule. ${ }^{8,9}$ This spectral shift cannot be completely attributed to dynamic stabilization, and it has been suggested that the interference of the primary and scattered waves in 
the molecular cage with singly scattered waves at neighboring molecules in clusters explains this experimental result. ${ }^{8,9}$ Furthermore, cluster size dependent spectral changes of the other resonances in the $\mathrm{S} 2 p$-excited $\mathrm{SF}_{6}$ have not been published before. Specifically, these are the $\mathrm{S} 2 p \rightarrow 6 \mathrm{a}_{1 \mathrm{~g}}$-transition below the $\mathrm{S} 2 p$ ionization energy and the $\mathrm{S} 2 p \rightarrow 4 \mathrm{e}_{\mathrm{g}}$-transition in the $\mathrm{S} 2 p$-continuum. ${ }^{15}$ This provides the motivation for the present work, in which we focus on these transitions. Cation yields of clusters are discussed along with yields from molecular fragments. The results are compared to previous work on the $\mathrm{S} 2 p \rightarrow 2 \mathrm{t}_{2 \mathrm{~g}}$-transition. ${ }^{8,9}$ The relationship between gasto-cluster shifts and changes in line shape of the continuum resonances is discussed in detail. The analysis is complicated by spin-orbit splitting of all resonances associated with $\mathrm{S} 2 p$ electrons excitation. The separation of both spin-orbit components is specifically complicated for the broad S $2 p \rightarrow 4 \mathrm{e}_{\mathrm{g}}$ bands, since they are strongly asymmetric in shape.

In this work, the double barrier optical potential (DBOP) model $^{8,9}$ is applied for separation of the spin-orbit components serving to assign spectral gas-to-cluster shifts. The line shapes of the individual spin-orbit-split components of the shape-resonances are described by using a convolution of Gaussians and symmetric and asymmetric Lorentzians. Mixed asymmetric Gaussian and Lorentzian functions have been previously used to describe line shapes in core-level photoelectron spectroscopy. ${ }^{18,19}$ Asymmetric Lorentzian functions evidently dominate the shape of the core-to-valence transitions above the ionization threshold and account for the temporal trapping of the photoelectron within the finite size molecular and cluster potentials. This approach allows us to distinguish the spectral gas-to-cluster shifts from the gas-tocluster changes in spectral distribution of oscillator strength (SDOS) for $\mathrm{S} 2 p_{3 / 2}$ and $2 p_{1 / 2} \rightarrow 2 \mathrm{t}_{2 \mathrm{~g}}$ - and $4 \mathrm{e}_{\mathrm{g}}$-transitions. The shifts and changes in the Lorentzian and Gaussian widths as well as the asymmetry parameters of the shape resonances are discussed in terms of the intermolecular interference of the primary and scattered photoelectron waves. The present results are shown to give clear evidence that the asymmetric Lorentzian distribution dominates in the line shape of core-tovalence transitions above the ionization threshold and interference of the primary and scattered waves within the molecular cage with singly scattered waves at neighboring molecules determines the gas-to-cluster shift.

\section{EXPERIMENTAL}

The experimental setup is similar to previous work. $3,8,9$ The present results cover, however, a significantly wider energy range since the undulator based photon source has become fully tunable, which was not possible in our previous work on $\mathrm{SF}_{6}$ clusters. ${ }^{8,9}$ This provides novel information on the $\mathrm{S} 2 p \rightarrow 6 \mathrm{a}_{1 \mathrm{~g}}$-resonance below the edge as well as the $\mathrm{S} 2 p \rightarrow 4 \mathrm{e}_{\mathrm{g}}$-shape resonance. Briefly, neat $\mathrm{SF}_{6}$ is expanded through a $50 \mu \mathrm{m}$ nozzle at room temperature at a stagnation pressure of 5 bars. No cluster formation is observed under these conditions. Variable size $\mathrm{SF}_{6}$-clusters are produced by the expansion of a mixture of $10 \% \mathrm{SF}_{6}$ in argon at variable temperature. The stagnation pressure is kept at 5 bars during the experiments. The gases are of commercial quality $\left(\mathrm{SF}_{6}\right.$ : Westfalen-Gas; Ar: Messer-Griesheim; purity >99.99\%).

The gas jet is crossed by a beam of monochromatic synchrotron radiation from the electron storage ring BESSY II (HZB Berlin, Germany) using the U49-SGM-beam line. The experiments are carried out with an energy resolving power $\mathrm{E} / \Delta \mathrm{E} \gg 5 \times 10^{3} .{ }^{8,9}$ The pressure in the ionization region is of the order of $10^{-5}$ mbar providing collision free conditions. Cations are detected by a time-of-flight mass spectrometer. Yields of mass-selected cations are taken by selecting a mass channel, while scanning the photon energy. Previous work already indicated that the yield of $\mathrm{SF}_{2}{ }^{++}(\mathrm{m} / \mathrm{z}=35)$ is exclusively formed via molecular fragmentation. ${ }^{8,9}$ Clusterspecific properties are obtained from the yield of $\mathrm{SF}_{6} \cdot \mathrm{SF}_{5}{ }^{+}$ $(\mathrm{m} / \mathrm{z}=273)$ (see Fig. 1). Cation yields of both channels are taken simultaneously in order to determine even small energy shifts at the $\mathrm{S} 2 p$-edges, similar to previous work. ${ }^{8,9}$ Note that the selected fragment $\mathrm{SF}_{6} \cdot \mathrm{SF}_{5}{ }^{+}$can either be formed via single or dissociative double ionization. Therefore, this fragment is suitable to probe electronic properties of all clusters decaying into this mass channel. We expect that all clusters below the critical size of dissociative double ionization, which is known to be $\left(\mathrm{SF}_{6}\right)_{\mathrm{n}}$ with $\mathrm{n} \leq 39,{ }^{20}$ can decay into the $\mathrm{SF}_{6} \cdot \mathrm{SF}_{5}{ }^{+}$-channel. Therefore, we assume that only electronic properties of small $\mathrm{SF}_{6}$ clusters are probed. The cation yields are normalized to the incident photon flux by using either the photocurrent, a gold mesh, or the total ion yield of rare gases. The photon energy scale is calibrated by comparison with high-resolution electron energy loss data. ${ }^{21}$ The results on the isolated molecule near the $\mathrm{S} 2 p$-ionization threshold are in full agreement with results published by Hudson et al..$^{15}$

\section{RESULTS}

Figure 1 displays an overview of the photoion yield of free $\mathrm{SF}_{6}$ (recorded as $\mathrm{SF}_{2}{ }^{++}$, cf. Fig. 1(a)) and $\mathrm{SF}_{6}$-clusters (recorded as $\mathrm{SF}_{6} \cdot \mathrm{SF}_{5}{ }^{+}$, cf. Fig. 1(b)) near the $\mathrm{S} 2 p$-edges. These high resolution measurements have been taken between $170 \mathrm{eV}$ and $210 \mathrm{eV}$, indicating that the changes upon cluster formation are in general small. This is in agreement with earlier low resolution work on molecular and solid $\mathrm{SF}_{6}{ }^{12,13}$ No spectral shift between the molecular and cluster spectrum is identified for the $\mathrm{S} 2 p \rightarrow 6 \mathrm{a}_{1 \mathrm{~g}}$-band and the band shape remains unchanged with an accuracy of $\Delta \mathrm{E}= \pm 3 \mathrm{meV}$ for this pre-edge valence transition. However, a spectral redshift upon cluster formation is observed for the $\mathrm{S} 2 p \rightarrow 2 \mathrm{t}_{2 \mathrm{~g}}$ - and $\mathrm{S} 2 p \rightarrow 4 \mathrm{e}_{\mathrm{g}}$-transitions. These are not distinguishable for the spin-orbit components of the $\mathrm{S} 2 p \rightarrow 2 \mathrm{t}_{2 \mathrm{~g}}$-band indicating a spectral redshift of $\Delta \mathrm{E}=35 \pm 5 \mathrm{meV}$, which is similar to previous work. ${ }^{8}$ For the $\mathrm{S} 2 p \rightarrow 4 \mathrm{e}_{\mathrm{g}}$-transitions, we observe a redshift by $\Delta \mathrm{E}=105 \pm 5 \mathrm{meV}$. This spectral shift refers to the maximum of the $4 \mathrm{e}_{\mathrm{g}}$-band for molecules and clusters. This is shown in the inset of Fig. 1, in which only a small region near the maximum of the band is displayed. Note that this shift is extracted for clarity from a fit of the experimental results (see details below). Asymmetric Lorentzian functions are used as mathematical models that fit the experimental data 


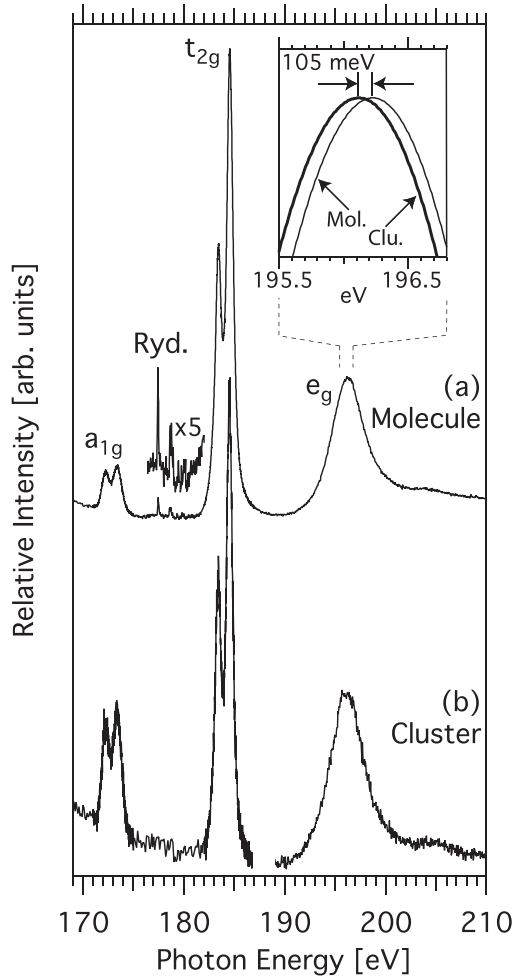

FIG. 1. Experimental $\mathrm{S} 2 p$-photoion yields of free $\mathrm{SF}_{6}$ (a) and clustered $\mathrm{SF}_{6}$ (b). The inset shows the redshift of the $\mathrm{S} 2 p \rightarrow 4 \mathrm{e}_{\mathrm{g}}$-band of cluster (Clu.) relative to the molecular transition (Mol.) by $105 \pm 5 \mathrm{meV}$ (see text for further details).

near the $\mathrm{S} 2 p \rightarrow 4 \mathrm{e}_{\mathrm{g}}$-transition. This is explained in detail below (see Sec. III A).

\section{A. Model calculations: SDOS for core-to-valence transitions embedded in continua}

The molecular origin of the $2 \mathrm{t}_{2 \mathrm{~g}}$-resonance in $\mathrm{SF}_{6^{-}}$ clusters has been fully explained by the DBOP model, as outlined before. ${ }^{8,9}$ The internal potential barrier, formed by the pseudopotential of the fluorine octahedron and the centrifugal repulsion, ${ }^{8,22}$ plays a double role in clusters. It provides, on the one hand, an effective trap for the $\mathrm{S} 2 p$-photoelectron inside the molecular cage caused by the electronegative fluorine. On the other hand, it shields the inner-well states from incoming electrons, which are backscattered from neighboring $\mathrm{SF}_{6}$ moieties in clusters. The interplay of the trapping and shielding properties of the molecular cage controls the gas-tocluster redshift of the $\mathrm{S} 2 p \rightarrow 2 \mathrm{t}_{2 \mathrm{~g}}$-transitions.

The gas-to-cluster shift has been determined by using Voigt functions in order to de-convolute the experimental results. ${ }^{8,9}$ Voigt functions are in general not the most appropriate way to model shape resonances. This is due to their asymmetry, which is a consequence of spectral variations of the trapping and shielding properties of the electronegative fluorine and must be considered when the $\mathrm{S} 2 p \rightarrow 2 \mathrm{t}_{2 \mathrm{~g}}$ - and $\mathrm{S} 2 p \rightarrow 4 \mathrm{e}_{\mathrm{g}}$-transitions are analyzed.

In the framework of the quasiatomic approach, the interferential function $M_{\Gamma}(k)$ describes the SDOS for X-ray transi- tions in molecules and clusters according to

$$
M_{\Gamma}(k)=\operatorname{Re}[(\mathbf{1}+\mathbf{B S}) /(\mathbf{1}-\mathbf{B S})]_{l l^{\prime} \Gamma} .
$$

This function originates from intra- and intermolecular interferences of the primary and scattered photoelectron waves. $\mathbf{B}$ and $\mathbf{S}$ are the electron reflection and scattering matrices, $l$ is the photoelectron orbital momentum, $l^{\prime}$ is the orbital momentum of the reflected wave, $l=l^{\prime}$ in the vicinity of the S $2 p$-core hole, and $\Gamma$ is the irreducible representation of the point group symmetry of $\mathrm{SF}_{6} O_{h}$ (see for details Refs. 8 and 9). Expanding $M_{\Gamma}(k)$ in a Taylor series for small deviations of the photoelectron wavenumber $k-k_{0}$ at the resonance position $k_{0}$, and keeping only the quadratic term, since odd terms can be neglected, we derive

$$
M_{\Gamma}(k) \approx \frac{2 C_{m}}{W_{L}^{\Gamma}(k) R_{m}} \cdot \frac{1}{\left(1+\frac{4\left(k-k_{0}\right)^{2}}{\left(W_{L}^{\Gamma}(k)\right)^{2}}\right)} \equiv L_{\Gamma}^{\alpha}(k) .
$$

This expansion is valid for the spectral interval $\Omega_{\Gamma}\left(k_{0}\right)$, which is defined by the condition

$$
\frac{1}{3}\left(k-k_{0}\right)^{2} R_{m}^{2} \ll 1 .
$$

Without core hole lifetime broadening the full-width-at-the half-maximum (FWHM) $W_{L}^{\Gamma}$ is given by

$$
W_{L}^{\Gamma}(k)=\frac{\left(1-\left|B_{l \Gamma}(k)\right|\right)}{R_{m} \sqrt{\left|B_{l \Gamma}(k)\right|}} .
$$

In Eqs. (2)-(4), $B_{l \Gamma}$ is the amplitude of the backscattered photoelectron waves, $R_{m}$ is the interatomic $\mathrm{S}-\mathrm{F}$ distance in $\mathrm{SF}_{6}$, and the coefficient $C_{m}$ is equal to $\left(1+\left|B_{l}\left(k_{0}\right)\right|\right) / \sqrt{\left|B_{l}\left(k_{0}\right)\right|}$.

Equation (2) shows the Lorentzian profile $L_{\Gamma}^{\alpha}(k)$ of a shape resonance within the interval $\Omega_{\Gamma}\left(k_{0}\right)$. Since the FWHM is $k$-dependent, an asymmetry and redshift of $\max \left\{L_{\Gamma}^{\alpha}(k)\right\}$ from $k_{0}$ appears in the spectra. The observed position of the maximum is denoted by $k_{\max }$ in the following. When the module $\left|B_{l \Gamma}\right|$ is close to unity, the FWHM is so narrow that spectral variations of the amplitudes within $\Omega_{\Gamma}$ can be neglected. In the limiting case $W_{L}^{\Gamma} \rightarrow 0$, the quasi-discrete resonance shows an almost symmetric Lorentzian profile and can be successfully described by Voigt functions if the experimental broadening is taken into account, assuming a Gaussian distribution. This explains the successful application of Voigt functions to fit the narrow $2 \mathrm{t}_{2 \mathrm{~g}}$-shape resonances in $\mathrm{SF}_{6} .8,9,15$ When the molecular cage becomes more transparent and the module $\left|B_{l \Gamma}\right|$ decreases, the resonance becomes broader and more asymmetric in shape.

The direct use of the spectral distribution $L_{\Gamma}^{\alpha}$ for the analysis of the experimental spectra encounters difficulties with the complicated spectral dependence of $W_{L}^{\Gamma}$. This is circumvented by expanding the amplitudes $B_{l \Gamma}(k)$ for small displacements $k-k_{0}$. Assuming that the amplitudes do not vary quickly within $\Omega_{\Gamma}\left(k_{0}\right)$, we keep only the linear term in the expansion, so that

$$
W_{L}^{\Gamma}(k) \approx \frac{2 \cdot W_{L}^{\Gamma}\left(k_{0}\right)}{1+\exp \left[\alpha_{\Gamma}\left(k^{2}-k_{0}^{2}\right)\right]} .
$$


Thus, a single parameter $\alpha_{\Gamma}$ specifies the asymmetry of the shape resonance

$$
\left.\alpha_{\Gamma} \approx \frac{1}{4 k\left|B_{l \Gamma}\right|} \cdot \frac{1+\left|B_{l \Gamma}\right|}{1-\left|B_{l \Gamma}\right|} \cdot \frac{\partial\left|B_{l \Gamma}\right|}{\partial k}\right|_{k=k_{0}} .
$$

Assuming that $\left|B_{l \Gamma}\right|(k)$ drops monotonously from unity at $k=0$ to zero at $k \rightarrow \infty$ implies that the line asymmetry $\alpha_{\Gamma}$ must be negative.

As a second step, we introduce the model distribution

$$
\tilde{M}_{\Gamma}(k) \approx \iint M_{\Gamma}\left(k^{\prime}\right) L_{2 p}\left(k-k^{\prime}\right) G\left(k^{\prime}-k^{\prime \prime}\right) d k^{\prime} d k^{\prime \prime}
$$

of the oscillator strength for core-to-valence transitions in the continuum. Within $\Omega_{\Gamma}\left(k_{0}\right)$ we substitute $M_{\Gamma}\left(k^{\prime}\right) \rightarrow L_{\Gamma}^{\alpha}\left(k^{\prime}\right)$ (cf. Eq. (2)). This gives

$$
\tilde{M}_{\Gamma}(k) \approx \iint L_{\Gamma}^{\alpha}\left(k^{\prime}\right) L_{2 p}\left(k-k^{\prime}\right) G\left(k^{\prime}-k^{\prime \prime}\right) d k^{\prime} d k^{\prime \prime} .
$$

The integral (7) is used in the following to analyze the experimental line shapes. It contains the following contributions to the overall line shape: (i) the asymmetric Lorentzian $L_{\Gamma}^{\alpha}(k)$ approximates the line shape of the quasi-bound state with respect to emission of the photoelectron; (ii) the symmetric Lorentzian $L_{2 p}\left(k-k^{\prime}\right)$ represents the $\mathrm{S} 2 p^{-1}$ core hole decay with natural line-width $W_{L}^{2 p}$; (iii) the Gaussian $G\left(k^{\prime}-k^{\prime \prime}\right)$ expresses the bandwidth $W_{\mathrm{G}}$ of the photon source. The model distribution $\tilde{M}_{\Gamma}(k)$ is applicable to shape resonances under conditions in which the widths $W_{L}^{2 p}$ and $W_{\mathrm{G}}$ are significantly narrower than $\Omega_{\Gamma}\left(k_{0}\right)$. This is evidently the case for the experimental $2 \mathrm{t}_{2 \mathrm{~g}}$ - and $4 \mathrm{e}_{\mathrm{g}}$-resonances.

\section{B. Experimental data analysis}

As a next step, the distribution $\tilde{M}_{\Gamma}(k)$ is used to fit the experimental spectra and to decompose the spin-orbit-split transitions $\mathrm{S} 2 p \rightarrow 2 \mathrm{t}_{2 \mathrm{~g}}$ and $4 \mathrm{e}_{\mathrm{g}}$. The experimental and model $\mathrm{S}$ $2 p_{3 / 2}$ and $\mathrm{S} 2 p_{1 / 2}$ spectra are shown in Fig. 2 for the $2 \mathrm{t}_{2 \mathrm{~g}}$ - and Fig. 3 for the $4 \mathrm{e}_{\mathrm{g}}$-resonances for both free and clustered $\mathrm{SF}_{6}$. A comparison between the modeled and experimental spectra shows a close resemblance. The widths $W_{L}^{t_{2 g}}$ and $W_{\mathrm{G}}$, the line asymmetry $\alpha_{t_{28}}$, as well as the intensity ratio of the spinorbit components are used as fit parameters. The widths of the $\mathrm{S} 2 p_{1 / 2}$ - and $\mathrm{S} 2 p_{3 / 2}$-components are assumed for simplification to be identical, while it is known that slight differences between both spin-orbit components exist for the $t_{2 g^{-}}$ resonance. ${ }^{15} W_{L}^{2 p}$ is referring to $\mathrm{S} 2 p^{-1}$ core hole decay and is assumed to be $0.22 \mathrm{eV}$. This value has been published previously, based on a Franck-Condon analysis of the $\mathrm{S} 2 p$ $\rightarrow \mathrm{a}_{1 \mathrm{~g}}$ transition. ${ }^{15}$ It is assumed in the following that $W_{L}^{2 p}$ is constant for all inner-well states.

The $\mathrm{S} 2 p \rightarrow 2 \mathrm{t}_{2 \mathrm{~g}}$-transitions have been investigated in previous work in greater detail. ${ }^{8,9,15}$ The $\tilde{M}_{t_{2 g}}(k)$-analysis shows that the model distribution is able to reproduce the experimental results within the interval $\Omega_{2 t_{2 g}} \approx 2.5 \mathrm{eV}$ around the shape resonance. The parameters of the $\mathrm{S} 2 p \rightarrow 2 \mathrm{t}_{2 \mathrm{~g}^{-}}$ resonances, as extracted from the experimental spectra by using the SDOS model, are compiled in Table I. The width of the shape resonance $W_{2 p}^{2 t_{2 g}}$, corresponding to both the

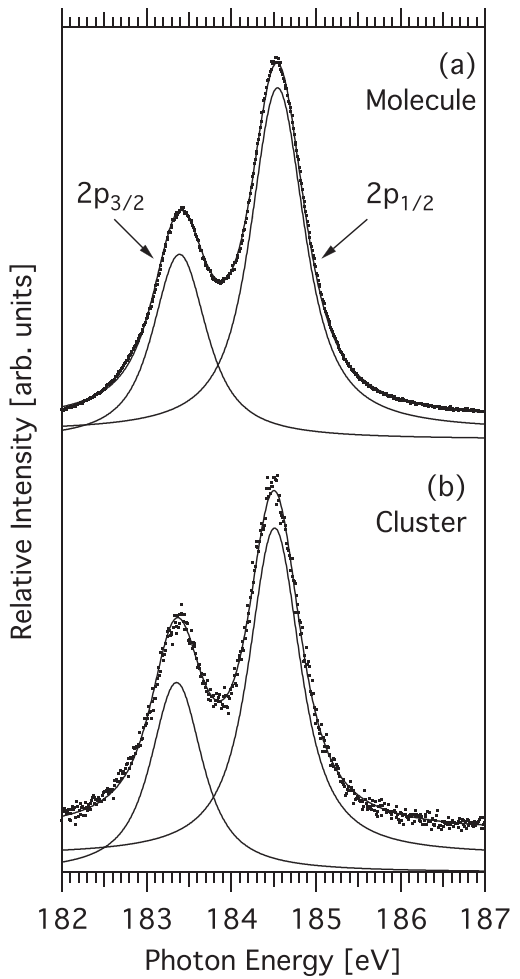

FIG. 2. The de-convoluted spin-orbit splitting of the $S 2 p_{3 / 2,1 / 2} \rightarrow 2 \mathrm{t}_{2 \mathrm{~g}}$ transitions in molecular $\mathrm{SF}_{6}$ (a) and $\mathrm{SF}_{6}$-clusters (b). Experimental data are shown as dots. Solid lines represent results of fit functions containing contributions from asymmetric Lorentzians (cf. Eq. (7)).

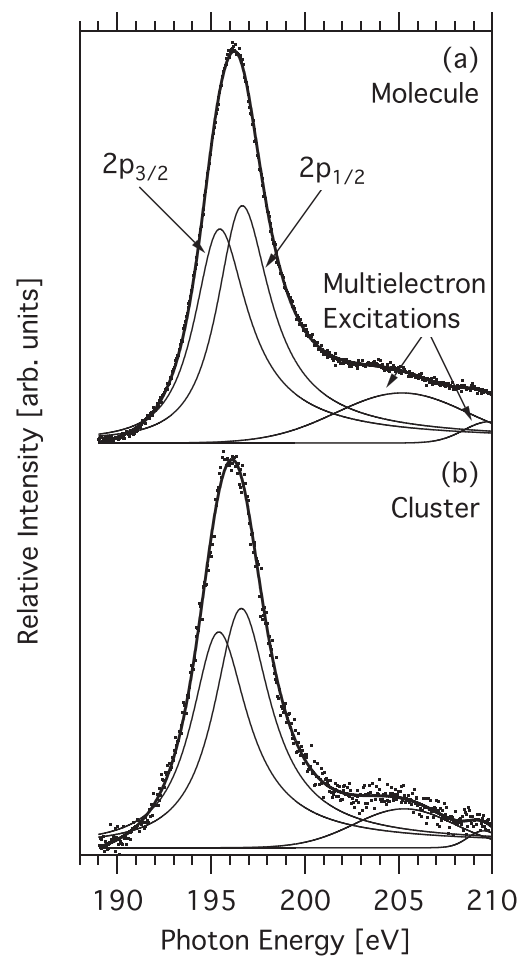

FIG. 3. Spectral de-convolution of the $\mathrm{S} 2 p_{1 / 2,3 / 2} \rightarrow 4 \mathrm{e}_{\mathrm{g}}$-transition in molecular $\mathrm{SF}_{6}$ (a) and $\mathrm{SF}_{6}$-clusters (b). Experimental data are shown as dots. Solid lines represent results of fit functions containing contributions from asymmetric Lorentzians (cf. Eq. (7)). 
TABLE I. Spectral parameters of the individual spin-orbit split S $2 p_{3 / 2,1 / 2}$ components of the shape resonances in free and clustered $\mathrm{SF}_{6}$ extracted from the experimental data analysis. Energy positions derived from the experimental results $\left(E_{\max }\right)$, maxima of the spectral distribution $\left(E_{0}\right)$, Lorentzian and Gaussian FWHM $\left(W_{2 p}^{\Gamma}\right)$ and $\left(W_{\mathrm{G}}\right)$, Lorentzian FWHM referring to $2 p$-hole decay and the photoelectron trapping time within the molecular cage are $W_{L}^{2 p}$ and $W_{L}^{\Gamma}$, respectively. A convolution of a symmetric and asymmetric Lorentzian distribution is used in order to approximate the width $W_{2 p}^{\Gamma}$. The superscript and the subscript $\Gamma$ refer to $t_{2 g}$ and $e_{g}$ denoting the shape resonances under study and the line asymmetry is denoted by $\alpha_{\Gamma}$. The error of the energy positions and the line asymmetry is estimated be $\pm 5 \mathrm{meV}$ and $\pm 0.1 \mathrm{eV}^{-1}$, respectively; the error of the widths $W_{2 p}^{\Gamma}$ and $W_{L}^{\Gamma}$ for $2 \mathrm{t}_{2 \mathrm{~g}}$ - and $4 \mathrm{e}_{\mathrm{g}}$-shape resonances is estimated be $\pm 10 \mathrm{meV}$ and $\pm 150 \mathrm{meV}$, respectively. See text for further details.

\begin{tabular}{|c|c|c|c|c|c|c|c|c|c|}
\hline & $\begin{array}{c}E_{\max }, \mathrm{S} 2 p_{3 / 2} \\
(\mathrm{eV})\end{array}$ & $\begin{array}{c}E_{\max }, \mathrm{S} 2 p_{1 / 2} \\
(\mathrm{eV})\end{array}$ & $\begin{array}{c}E_{0}, \mathrm{~S} 2 p_{3 / 2} \\
(\mathrm{eV})\end{array}$ & $\begin{array}{c}E_{0}, \mathrm{~S} 2 p_{1 / 2} \\
(\mathrm{eV})\end{array}$ & $\begin{array}{l}W_{L}^{2 p} \\
(\mathrm{eV})\end{array}$ & $\begin{array}{l}W_{L}^{\Gamma} \\
(\mathrm{eV})\end{array}$ & $\begin{array}{l}W_{2 p}^{\Gamma} \\
(\mathrm{eV})\end{array}$ & $\begin{array}{l}W_{\mathrm{G}} \\
(\mathrm{eV})\end{array}$ & $\begin{array}{c}\alpha_{\Gamma} \\
\left(\mathrm{eV}^{-1}\right)\end{array}$ \\
\hline Molecular $2 \mathrm{t}_{2 \mathrm{~g}}$ & 183.387 & 184.544 & 183.393 & 184.547 & 0.22 & 0.386 & 0.61 & 0.32 & -0.15 \\
\hline Clustered $2 \mathrm{t}_{2 \mathrm{~g}}$ & 183.352 & 184.509 & 183.353 & 184.507 & 0.22 & 0.372 & 0.6 & 0.29 & -0.02 \\
\hline Molecular $4 \mathrm{e}_{\mathrm{g}}$ & 195.465 & 196.665 & 195.713 & 196.867 & 0.22 & 3.36 & 3.63 & 0.58 & -0.3 \\
\hline Clustered $4 \mathrm{e}_{\mathrm{g}}$ & 195.413 & 196.612 & 195.563 & 196.717 & 0.22 & 3.41 & 3.69 & 0.58 & -0.14 \\
\hline
\end{tabular}

photoelectron trapping time and the core-hole lifetime is also compiled in Table I.

$E_{\max }$ is the energy position of the maximum and $E_{0}$ is the energy position of the shape resonance, corresponding to $k=k_{0}$. The position of the intensity maximum $E_{\max }$ cannot be immediately determined from the experimental spectra with sufficient accuracy. This is due to the experimental signal-to-noise ratio near the maxima. $E_{\max }$ is determined as follows: (i) in the case of the $2 \mathrm{t}_{2 \mathrm{~g}}$-resonance, the maximum of the fit functions $L_{\Gamma}^{\alpha}(k)$ are used for both spin-orbit components (cf. Eq. (2)), since these are well separated; and (ii) the spin-orbit components of the S $2 p_{3 / 2} \rightarrow 4 \mathrm{e}_{\mathrm{g}}$ - and $\mathrm{S} 2 p_{1 / 2}$ $\rightarrow 4 \mathrm{e}_{\mathrm{g}}$-transitions are blended. Therefore, the corresponding fit functions $L_{\Gamma}^{\alpha}(k)$ are added and the maximum of the resulting curve is used for determining the energy positions of the spin-orbit components.

The extracted Gaussian widths are found to vary slightly for the free molecule and clusters. This variation can be understood, if we take into account that $W_{\mathrm{G}}$ specifies not only the photon source but the inhomogeneous broadening of the quasi-bound states, which is a result of the effective quadratic intra- and intermolecular displacements. ${ }^{9}$ Besides, the extracted Gaussian widths $W_{\mathrm{G}}$ are influenced by multielectron and vibrational excitations. These effects will be discussed below in greater detail. The intensity ratio of the $\mathrm{S} 2 p_{3 / 2}$ and $2 p_{1 / 2}$-components is found to be equal to 0.9 , which is different from the statistical limit for $j j$ coupling. It has been stated previously that this intensity ratio indicates that the exchange interaction between the excited electron and the core-hole is comparable to the spin-orbit interaction for innerwell states. ${ }^{15,16}$ The ratio is also dependent on the assumption that the widths of the $\mathrm{S} 2 p_{1 / 2}$ and $\mathrm{S} 2 p_{3 / 2}$-components are identical.

In general, the spectroscopic characteristics of the $\mathrm{S} 2 p_{3 / 2}$ and $2 p_{1 / 2} \rightarrow 2 \mathrm{t}_{2 \mathrm{~g}}$-transitions are in full agreement with the results obtained from our previous work. ${ }^{8,9}$ However, the present model is improved, since the mean quadratic deviation between the experimental and modeled spectra is reduced nearly $20 \%$ in comparison to a pure Voigt fit of the shape resonances. Therefore, the $\tilde{M}_{\Gamma}$ model is applied in the following in order to investigate the unresolved S $2 p_{3 / 2}$ and $2 p_{1 / 2} \rightarrow 4 \mathrm{e}_{\mathrm{g}}$ bands. We include only two components to fit the bands and assume that, similar to the $2 \mathrm{t}_{2 \mathrm{~g}}$-resonance, vibrational excitations do not play an essential role in their shape (see Sec. IV).
The experimental and modeled $\tilde{M}_{e_{g}}(k)$ spectra of the S $2 p \rightarrow 4 \mathrm{e}_{\mathrm{g}}$-shape resonance are plotted in Fig. 3. There is good agreement within the interval $\Omega_{4 e_{g}} \approx 10 \mathrm{eV}$. Note that the $\mathrm{S} 2 p_{3 / 2}$ and S $2 p_{1 / 2}$-spin-orbit components of the broad bands have been successfully de-convoluted by using the asymmetric $\tilde{M}_{e_{g}}(k)$ distributions. The extracted parameters of the spin-orbit split $\mathrm{S} 2 p_{3 / 2}$ and $\mathrm{S} 2 p_{1 / 2} \rightarrow 4 \mathrm{e}_{\mathrm{g}}$-components are also listed in Table I. The present results indicate for the $\mathrm{S}$ $2 p_{3 / 2}$ and $\mathrm{S} 2 p_{1 / 2} \rightarrow 4 \mathrm{e}_{\mathrm{g}}$-transitions a substantial asymmetry in the Lorentzian profiles, where it is found that $W_{L}^{e_{g}} \gg W_{\mathrm{G}}$ and $W_{L}^{e_{g}} \gg W_{L}^{2 p}$. The spectral redshift $\Delta E_{0}$ is found to be $150 \pm 5 \mathrm{meV}$. This differs from the experimental value, corresponding to $105 \pm 5 \mathrm{meV}$, as derived from a summation over both spin-orbit components of the unresolved band shown in Fig. 3(b).

According to the present analysis, the asymmetric Lorentzian distribution $L_{\Gamma}^{\alpha}(k)$ controls the shape of the spinorbit components of the $\mathrm{S} 2 p \rightarrow 2 \mathrm{t}_{2 \mathrm{~g}}, 4 \mathrm{e}_{\mathrm{g}}$-bands, as shown in Figs. 2 and 3. This supports the assignment that the bands are due to shape resonances. The substantial broadening of the spin-orbit components of the $4 \mathrm{e}_{\mathrm{g}}$-bands relative to the $2 \mathrm{t}_{2 \mathrm{~g}}$ bands is conditioned by the increase of the Lorentzian widths $W_{L}^{\Gamma}$.

By using the uncertainty relationships $W_{L}^{\Gamma} \tau^{\text {trap }}{ }_{\Gamma}>\hbar$ and $W_{2 p}^{\Gamma} \tau_{\Gamma}>\hbar$, we determine, respectively, the photoelectron trapping time $\tau^{\text {trap }} \Gamma$ and the lifetime $\tau_{\Gamma}$ of the shape resonances. The derived lifetimes $\tau_{2 t_{2 g}} \approx 1.7 \mathrm{fs}$ and $\tau_{4 e_{g}} \approx 0.2 \mathrm{fs}$ are mainly limited by the photoelectron trapping time $\tau_{2 t_{2 g}}^{\text {trap }}$ $\approx 1.1 \mathrm{fs}$ and $\tau_{4 e_{g}}^{t r a p} \approx 0.2 \mathrm{fs}$. These are considerably shorter than the S $2 p$-core-hole lifetime of the inner-well states of $\approx 3$ fs.

The observed energy $E_{\max }$ of the maxima of SDOS for the above discussed transitions is shifted to lower energy relative to the resonance positions $E_{0}$. This displacement $E_{0}-E_{\max }$ is $\approx 5 \mathrm{meV}$ and $\approx 205 \mathrm{meV}$, respectively, for the $2 \mathrm{t}_{2 \mathrm{~g}}$ - and $4 \mathrm{e}_{\mathrm{g}}$-resonances in free $\mathrm{SF}_{6}$, whereas values of $\approx 0 \mathrm{meV}$ and $\approx 127 \mathrm{meV}$ are derived for these resonances in $\mathrm{SF}_{6}$-clusters. This is due to the decrease in line asymmetry in clusters (see Table I). As a consequence of this asymmetry, the observed gas-to-cluster redshift of the maxima: $\Delta E_{\max }=E_{\max }$ [cluster] $-E_{\max }[\mathrm{mol}.] \approx 35 \pm 5 \mathrm{meV}$ coincides precisely with the reported value in our previous work. ${ }^{8,9}$ It differs, however, from the redshift of the $2 \mathrm{t}_{2 \mathrm{~g}}$-resonance positions $\Delta E_{0}=E_{0}$ [cluster] 
$-E_{0}$ [mol.] $\approx 40 \pm 5 \mathrm{meV}$. Similar results are derived for the $4 \mathrm{e}_{\mathrm{g}}$-resonance, in which the observed redshift $\Delta E_{\max }$ $\approx 105 \pm 5 \mathrm{meV}$ (cf. Fig. 1) differs from the resonance position difference $\Delta E_{0} \approx 150 \pm 5 \mathrm{meV}$.

\section{Spectral gas-to-cluster changes}

In the following, the gas-to-cluster changes regarding the spectral position and line shape of the $2 \mathrm{t}_{2 \mathrm{~g}}$ - and $4 \mathrm{e}_{\mathrm{g}}-$ resonances are considered, as presented in Table I. The DBOP model $^{8}$ is used to link the changes with intra- and intermolecular interferences of the photoelectron waves. For $\mathrm{SF}_{6}$ clusters, the internal barrier is sufficiently high to provide an effective trap for the S $2 p$-photoelectrons with a kinetic energy $K<20 \mathrm{eV}$ within the molecular cage. On the other hand, the external barrier is low, providing mainly a source for single scattering of the photoelectron in core-excited clusters. ${ }^{8,9}$ These characteristics of the DBOP refer to the weak cluster effect on the quasi-bound molecular states. In this case for the amplitude $B=|B| \exp \left(2 \mathrm{i} k R_{m}+\varphi\right)$ of electron waves backscattered from the double barrier, we derive

$$
\begin{gathered}
|B| \approx\left|B_{m}\right|+\overbrace{D\left|B_{c}\right| \cos 2 \vartheta}^{2^{\prime}}, \\
\tan \varphi=\tan \varphi_{m}-\overbrace{D \cdot \frac{\left|B_{c}\right| \sin 2 \vartheta}{\left|B_{m}\right| \cos ^{2} \varphi_{m}}}^{2^{\prime \prime}} .
\end{gathered}
$$

The subscripts $m$ (molecular) and $c$ (cluster) describe the amplitudes referring to the internal and external barriers, $\vartheta=k\left(R_{c}-R_{m}\right)+\varphi_{m}+\varphi_{c}, R_{c}$ is the intermolecular separation in clusters, and $D$ is the shielding factor. ${ }^{9}$ The subscripts $l$ and $\Gamma$ are omitted in the following.

The second terms on the right hand side of Eqs. (8) and (9), named $2^{\prime}$ and $2^{\prime \prime}$, specify the cluster effect on the inner-well states. These terms are strongly suppressed if the transparency of the molecular cage is weak. Neglecting any changes in the $\mathrm{S}-\mathrm{F}$-distance in free and clustered $\mathrm{SF}_{6}$ and assuming that $\left|\varphi-\varphi_{m}\right|=1$, one obtains

$$
\Delta E_{0} \approx C_{m} W_{L, m}^{\Gamma}\left|B_{c}\right| \sin 2 \vartheta .
$$

The Lorentzian width $W_{L}^{\Gamma}$ and the line asymmetry $\alpha_{\Gamma}$ are also subject of the gas-to-cluster change. Using Eqs. (4), (6) and (8), we derive for $\Delta W_{L}^{\Gamma}$ and $\Delta \alpha_{\Gamma}$

$$
\begin{aligned}
& \Delta W_{L}^{\Gamma} \approx-\frac{W_{L, m}^{\Gamma}}{2} \frac{\left|B_{c}\right|}{\left|B_{m}\right|}\left(1+\left|B_{c}\right|^{2}\right) \cos 2 \vartheta, \\
& \Delta \alpha_{\Gamma} \propto\left(-2 \frac{\partial\left|B_{m}\right|}{\partial k}\left|B_{c}\right|+D \frac{\partial\left|B_{c}\right|}{\partial k}\right) \cos 2 \vartheta \\
& \quad-2\left(R_{c}-R_{m}\right) D\left|B_{c}\right| \sin 2 \vartheta .
\end{aligned}
$$

Equations (10)-(12) link the gas-to-cluster changes in coreto-valence transitions with the molecular and cluster properties. Both, $\Delta E_{0}$ and $\Delta W_{L}^{\Gamma}$ are proportional to the Lorentzian width $W_{L, m}^{\Gamma}$ of the molecular shape resonances and the module $\left|B_{c}\right|$ of singly scattered waves at the neighboring molecules in clusters. In addition, the gas-to-cluster changes are also dependent on $\vartheta$. In dependence of the phase shift regarding both, spectral red- and blueshifts as well as narrowing and broadening of the molecular-like shape resonances are in general allowed in clusters.

The extracted parameters of the core-to-valence transitions (see Table I) agree with the relationships supporting the quasiatomic treatment of the shape resonance phenomena in free and clustered $\mathrm{SF}_{6}$. One clearly sees that both $\Delta E_{0}$ and $\Delta W_{L}^{\Gamma}$ are tightly related to the molecular Lorentzian width. Indeed, both the smallest shift $\approx 40 \mathrm{meV}$ and the change in Lorentzian width $\left|\Delta W_{L}^{2 t_{2 g}}\right| \approx 10 \mathrm{meV}$ are observed for the narrow $2 \mathrm{t}_{2 \mathrm{~g}}$-shape resonance. However, both the biggest redshift $\Delta E_{0} \approx 150 \mathrm{meV}$ and $\left|\Delta W_{L}^{4 e_{g}}\right| \approx 60 \mathrm{meV}$ refer to the broad $4 \mathrm{e}_{\mathrm{g}}$-shape resonance. Furthermore, both narrowing (for $2 \mathrm{t}_{2 \mathrm{~g}}$ ) and broadening (for $4 \mathrm{e}_{\mathrm{g}}$ ) effects of the Lorentzian widths are observed. Finally, there are substantial gas-tocluster changes in the line asymmetry.

\section{DISCUSSION}

The present high-resolution studies of free molecular $\mathrm{SF}_{6}$-clusters at the $\mathrm{S} 2 p$ photoionization threshold highlight both, the close resemblance and the distinct difference in SDOS for the $\mathrm{S} 2 p$-to-valence-transitions relative to the gas phase spectrum. No gas-to-cluster spectral shift is observed for the $6 \mathrm{a}_{1 \mathrm{~g}}$-band and this pre-edge feature remains entirely unchanged in shape upon cluster formation which is similar to other molecular cluster systems, in which only minor redshifts were determined. ${ }^{7}$ The spectral gas-to-cluster changes only observed for the $\mathrm{S} 2 p_{1 / 2,3 / 2} \rightarrow 2 \mathrm{t}_{2 \mathrm{~g}}$ - and $4 \mathrm{e}_{\mathrm{g}}$-transitions, which are embedded into the $\mathrm{S} 2 p$-ionization continua. These changes consist mainly of a spectral redshift as well as a specific redistribution in oscillator strength for these transitions. The analysis of the gas-to-cluster changes is complicated by spin-orbit splitting and the substantial asymmetry in their spectral shape. Voigt fitting is often used for pre-edge core-to-valence transitions, but this is not applicable to the transitions located above the $\mathrm{S} 2 p$-edge.

The quasiatomic approach is applied to investigate the S $2 p_{3 / 2,1 / 2} \rightarrow 2 \mathrm{t}_{2 \mathrm{~g}}$ - and $4 \mathrm{e}_{\mathrm{g}}$-transitions and to identify the spin-orbit components. Examining the interference of the primary and scattered waves we have revealed that the asymmetric Lorentzian distribution $L_{\Gamma}^{\alpha}(k)$ specifies the core-tovalence transitions, which are embedded into the continua, within the spectral interval $\Omega_{\Gamma}\left(k_{0}\right)$. For a comparison with the experimental data, we introduce the model distribution $\tilde{M}_{\Gamma}(k)$ as a convolution $L_{\Gamma}^{\alpha} \cdot L_{2 p} \cdot G$ of asymmetric and symmetric Lorentzian and Gaussian distributions and apply this model to the experimental spectra in the vicinity of the shape resonances. In contrast to Voigt functions, the model function $\tilde{M}_{\Gamma}(k)$ takes in addition to the core-hole decay and the spectral band width of the photon source also the temporary trapping of the photoelectron within the molecular cage into account. Assuming that the spectral characteristics of the core-hole decay and the photon source are known, this approach allows us to investigate if the asymmetric Lorentzian distribution $L_{\Gamma}^{\alpha}(k)$ controls the experimental spectra. If $L_{\Gamma}^{\alpha}(k)$ dominates the shape of the bands, we are able to decompose the spin-orbit components of the experimental bands, so that we can specify the photoelectron trapping within the 
molecular cage. On the other hand, if the experimental spectra cannot be reasonably fitted by $L_{\Gamma}^{\alpha}(k)$, we have the clear indication that there are other mechanisms active. In particular, if the bands contain substantial contributions from valence transitions, the experimental spectra cannot be reasonably reproduced as the energy distribution of the valence shells is not taken into account by this model.

The performed analysis of the $S 2 p$ photoabsorption spectra at the $2 \mathrm{t}_{2 \mathrm{~g}}$ - and $4 \mathrm{e}_{\mathrm{g}}$-shape resonances has demonstrated that the $\tilde{M}_{\Gamma}(k)$-distribution allows for the precise modeling of the experimental results and the de-convolution of the spinorbit components in free $\mathrm{SF}_{6}$ and in $\mathrm{SF}_{6}$-clusters. The asymmetric Lorentzian $L_{\Gamma}^{\alpha}(k)$ controls the experimental SDOS for the core-to-valence in the free molecule and clusters. Inspecting the extracted parameters, we note that the gas-to-cluster changes are tightly related with the $L_{\Gamma}^{\alpha}(k)$ variations. This implies that the cluster potential affects mainly the unoccupied $2 \mathrm{t}_{2 \mathrm{~g}}$ - and $4 \mathrm{e}_{\mathrm{g}}$-orbitals.

The substantial broadening of the $4 \mathrm{e}_{\mathrm{g}}$-resonances relative to the $2 \mathrm{t}_{2 \mathrm{~g}}$-resonances is completely determined by the increase in the Lorentzian width $W_{L, m}^{\Gamma}$. This is due to an increase in transparency of the molecular cage for fast photoelectrons as, according to Eq. (4), $W_{L, m}^{\Gamma}$ increases when $\left|B_{l \Gamma}\right|$ decreases. Using the extracted widths $W_{L}^{\Gamma}$, we estimate the photoelectron trapping time $\tau^{\text {trap }} \Gamma_{\Gamma}$ to be 1.1 fs for the $2 \mathrm{t}_{2 \mathrm{~g}}$ and 0.2 fs for the $4 \mathrm{e}_{\mathrm{g}}$-quasi-bound states in molecular $\mathrm{SF}_{6}$. Long trapping times give rise to the resonant character of the Auger decay, originating from S $2 p \rightarrow 2 \mathrm{t}_{2 \mathrm{~g}}$ - and S $2 p \rightarrow 4 \mathrm{e}_{\mathrm{g}}$ transitions. Due to the difference in $\tau^{\text {trap }} \Gamma$ the Auger spectra are expected to undergo substantial changes. This expectation agrees with previous results. ${ }^{23}$ There, it was found that the Auger decay spectra measured at these resonances are not identical and there are some other core-excited states than $\mathrm{S}$ $2 p^{-1} .23$

The Auger decay and multielectron properties of the $\mathrm{S}$ $2 p^{-1} 2 \mathrm{t}_{2 \mathrm{~g}}$-state are influenced by photoelectron recapture

$$
\begin{gathered}
\mathrm{SF}_{6}+\mathrm{h} v \rightarrow \mathrm{SF}_{6}{ }^{+}\left(2 p^{-1}\right)+e_{p h o t o}^{-}(K) \\
\rightarrow \mathrm{SF}_{6}{ }^{* *}\left(2 p^{-1} \mathrm{~V}^{-1}{ }_{i} \mathrm{~V}^{1}{ }_{j} \mathrm{~V}^{1}{ }_{k}\right)
\end{gathered}
$$

Coupling of the charge transfer channel (13) with the direct photoionization channel $\mathrm{SF}_{6}+\mathrm{h} v \rightarrow \mathrm{SF}_{6}+\left(2 p^{-1}\right)$ $+e_{\text {photo }}^{-}(K)$ gives rise to the resonant anion yield at the $2 \mathrm{t}_{2 \mathrm{~g}}$-shape resonance in $\mathrm{S} 2 p$ excited $\mathrm{SF}_{6} \cdot{ }^{24}$ Thus, in addition to the $\mathrm{SF}_{6}+\left(2 p^{-1}\right)$ the "2-hole-2-electron" state, $\mathrm{SF}_{6}{ }^{* *}\left(2 p^{-1} \mathrm{~V}^{-1}{ }_{i} \mathrm{~V}_{j}^{1} \mathrm{~V}^{1}{ }_{k}\right)$ can be assigned to the other state that contributes to the Auger spectra. ${ }^{23}$

In contrast to the $2 \mathrm{t}_{2 \mathrm{~g}}$-resonance, the multielectron properties of the $4 \mathrm{e}_{\mathrm{g}}$-shape resonance are associated with the energy transfer of the photoelectron coming through the molecular barrier. The photoelectron-valence electrons (PEVE) interaction is known to be essential in the shape resonance vicinity. ${ }^{25-27}$ In the present case, coupling of the direct channel with the inelastic ones, according to

$$
\begin{aligned}
& \mathrm{SF}_{6}+\mathrm{h} v \rightarrow \mathrm{SF}_{6}{ }^{+}\left(2 p^{-1}\right)+e_{\text {photo }}^{-}(K) \\
& \rightarrow \mathrm{SF}_{6}{ }^{+*}\left(2 p^{-1} \mathrm{~V}^{-1}{ }_{i} \mathrm{~V}^{1}{ }_{j}\right)+e_{\text {sat }}^{-}\left(K^{\prime}\right),
\end{aligned}
$$

leads to an energy transfer to the valence shells and to the appearance of new satellites in the photoelectron spectra. The "2-hole-1-electron" state $\mathrm{SF}_{6}{ }^{+*}\left(2 p^{-1} \mathrm{~V}^{-1}{ }_{i} \mathrm{~V}_{j}{ }_{j}\right)$ in channel (14) is the other core-excited state that contributes to the Auger spectra. Though both resonances demonstrate remarkable multielectron properties, they are assigned to shape resonances, because these properties are conditioned by trapping of the primary photoelectron within the molecular cage. Measurements of Kitajima et ll $^{28}$ confirm this assignment as the $\mathrm{S} 2 p$ photoionization spectra of $\mathrm{SF}_{6}$ behave resonantly in the vicinity of the $\mathrm{S} 2 p_{3 / 2,1 / 2} \rightarrow 2 \mathrm{t}_{2 \mathrm{~g}}$ - and $4 \mathrm{e}_{\mathrm{g}}$-transitions. Such behavior, according to Ref. 29, provides a sensitive probe of this assignment.

The PEVE interaction and vibrational excitations are the main sources of deviations between the experimental and modeled spectra. Evidently, Eqs. (2) and (7) do not take these effects into account. We have examined the correlation satellite observed in $\mathrm{S} 2 p$ photoemission spectra of $\mathrm{SF}_{6}$ at a binding energy $189 \mathrm{eV}$ in order to assess the impact of the PEVE interaction on the $\mathrm{S} 2 p_{3 / 2,1 / 2} \rightarrow 4 \mathrm{e}_{\mathrm{g}}$-transition. ${ }^{30}$ The integral (7) is applied to model this feature. An intriguing resemblance of the spectroscopic characteristics of the satellite and the $4 \mathrm{e}_{\mathrm{g}}$-shape resonance is revealed. In spite of low resolution measurements, ${ }^{30}$ the $\mathrm{S} 2 p_{3 / 2^{-}}$and $2 p_{1 / 2}$-components of the satellite band are successfully extracted and it is found that the Lorentzian $L_{\Gamma}^{\alpha}(k)$ with $W_{L}^{s a t} \approx 3.3 \mathrm{eV}$ and $\alpha^{\text {sat }}$ $=-0.24 \mathrm{eV}^{-1}$ dominate their shape. The similarity of the extracted width and the line asymmetry of the $S 2 p_{3 / 2}$ - and $2 p_{1 / 2}$-components of the satellite and of $4 \mathrm{e}_{\mathrm{g}}$-shape resonance in Table I indicates that the PEVE interaction is rather essential and, on the other hand, it does not disturb noticeably the band shape in S $2 p$-absorption spectra. According to Ref. 25 significantly more substantial changes are expected to occur in S $2 p$-photoionization spectra, which is in full agreement with previous results. ${ }^{28}$

Shape resonances are also known to produce non-FranckCondon distributions of vibrational excitations within the final ionic states. ${ }^{31,32}$ The present analysis is performed by assuming that the adiabatic $0 \rightarrow 0$-transition dominates in the core-to-valence transitions. This assumption is supported by earlier studies on the $\mathrm{S} 2 p_{3 / 2,1 / 2} \rightarrow 2 \mathrm{t}_{2 \mathrm{~g}}$-transitions. ${ }^{8,9,15}$ This is evidenced by inspecting deviations between the experimental results and the modeled $\tilde{M}_{2 t_{2 g}}(k)$ and $\tilde{M}_{4 e_{g}}(k)$ distributions. No evidence for highly vibrationally excited states is observed for the $\mathrm{S} 2 p_{3 / 2,1 / 2} \rightarrow 2 \mathrm{t}_{2 \mathrm{~g}}$-resonance, whereas the situation is more complicated for the $\mathrm{S} 2 p_{3 / 2,1 / 2} \rightarrow 4 \mathrm{e}_{\mathrm{g}}$-resonance.

The VDFN model ${ }^{32}$ is applied, taking the non-FranckCondon distribution of vibrational excitations into account for studying the vibrational excitations associated with the $\mathrm{S}$ $2 p_{3 / 2,1 / 2} \rightarrow 4 \mathrm{e}_{\mathrm{g}}$-transitions. A preliminary $\tilde{M}_{4 e_{g}}(k)$-analysis ${ }^{33}$ using the VDFN model has shown, that in addition to the adiabatic transition, which plays a leading role for the band shape, there is a weak contribution of the vibrationally excited $(\mathrm{v}=1)$ state. It was found that the relative intensity of the $\mathrm{S}$ $2 p_{3 / 2,1 / 2}(\mathrm{v}=0) \rightarrow 4 \mathrm{e}_{\mathrm{g}}(\mathrm{v}=1)$ is $\approx 0.1$ and the Lorentzian $L_{\Gamma}^{\alpha}(k)$ dominates in shape of the both vibrationally resolved $\mathrm{S} 2 p_{3 / 2,1 / 2}(\mathrm{v}=0) \rightarrow 4 \mathrm{e}_{\mathrm{g}}(\mathrm{v}=0,1)$-resonances. Note that extracted relative intensity pattern is in agreement with the highresolution $\mathrm{S} 2 p$ photoelectron spectra of $\mathrm{SF}_{6}$ recorded at a 
photon energy of $220 \mathrm{eV} .^{34}$ There it was found that the totally symmetric $0 \rightarrow 1$-vibrational transition can be identified with a relative intensity of 0.123 . Thus, the individual spinorbit components of the $4 \mathrm{e}_{\mathrm{g}}$-resonances in free and clustered $\mathrm{SF}_{6}$ can reasonably be approximated by the single integral (7), as shown in Figs. 2 and 3. Certainly, further experimental and theoretical studies are necessary to gain a more solid understanding of SDOS for core-to-valence transitions in free molecules and molecular clusters with respect to the occurrence of multielectron and vibrational effects on the core-tovalence transitions embedded in core ionization continua.

Within the DBOP model the gas-to-cluster changes in core-to-valence transitions are assigned to singly scattered photoelectrons from the quasi-bound molecular-like states in $\mathrm{SF}_{6}$-clusters, rather than to dynamic stabilization and sitespecific shifts due to intermolecular interactions. According to Eqs. (10) and (11), the gas-to-cluster effects are proportional to (i) the Lorentzian widths $W_{L, m}^{\Gamma}$ of the molecular shape resonances and (ii) the module $\left|B_{c}\right|$ of the backscattered electron waves at neighboring molecules in clusters. The analysis of the experimental data supports the occurrence of the cluster $W_{L, m}^{\Gamma}\left|B_{c}\right|$-effect (WB-effect). Considering this effect, even a size dependence of the gas-to-cluster changes can be predicted. This is due to the dependence of $\left|B_{c}\right|$ on the number of neighbors. Therefore, the gas-to-solid redshifts of the $2 \mathrm{t}_{2 \mathrm{~g}}$ and $4 \mathrm{e}_{\mathrm{g}}$-bands are expected to be larger than the gas-to-cluster shifts.

Analyzing the WB-effect, we derive $\left|B_{b}(k)\right| \approx 1-\frac{N_{s}}{N_{b}}$ $\left(1-\left|B_{c}(k)\right|\right)$ for $k \ll 1$ and $\left|B_{b}(k)\right| \approx \frac{N_{b}}{N_{s}}\left|B_{c}(k)\right|$ for $k \gg 1, B_{b}$ denotes the amplitude referring to the bulk sites and $\frac{N_{b}}{N_{s}}$ is the ratio of the nearest neighbors in bulk and surface sites, and $N_{b}=12$ for solid $\mathrm{SF}_{6}$. Thus, for the gas-to-solid redshift $\Delta E_{0}{ }^{\text {sol }}$, we derive

$$
\Delta E_{0}<\Delta E_{0}^{\text {sol }}<2 \Delta E_{0}
$$

which is consistent with the experimental results, considering that a larger difference $\Delta E_{0}{ }^{\text {sol }}-\Delta E_{0}$ is found for the $4 \mathrm{e}_{\mathrm{g}}$ resonance, as compared to the $2 \mathrm{t}_{2 \mathrm{~g}}$-resonance.

This WB-effect is expected to be rather general and applicable to core-to-valence-transitions in molecular solids as well as to core-to-valence-transitions, which are embedded into the conduction band. In solids, it can be studied, e.g., by the experimental X-ray absorption fine structure of $\mathrm{KPF}_{6}$ and $\mathrm{NH}_{4} \mathrm{PF}_{6}$ crystals containing chemically stable molecular-like moieties, whereby the experimental spectra have been reported before. ${ }^{35}$ The potential of the almost octahedral $\mathrm{PF}_{6}{ }^{-}$anions plays dominant role in forming the $\mathrm{P} 2 p$-to-valence transitions in solids and determines their strong similarity between the $\mathrm{S} 2 p$-tovalence transitions in molecular $\mathrm{SF}_{6}{ }^{36}$ In agreement with the present work, the experimental spectra on the mentioned solids ${ }^{36}$ do not show any spectral shift of the lowest $\mathrm{P} 2 p_{3 / 2,1 / 2} \rightarrow 6 \mathrm{a}_{1 \mathrm{~g}}$-transitions. There is a distinct shift by $\approx 0.2 \mathrm{eV}$ for the $2 \mathrm{t}_{2 \mathrm{~g}}$ - and $\approx 0.6 \mathrm{eV}$ for the $4 \mathrm{e}_{\mathrm{g}}$-transitions, as extracted for $\mathrm{KPF}_{6}$ in comparison with $\mathrm{NH}_{4} \mathrm{PF}_{6}$. These spectral shifts fully agree with Eq. (10) and support the applicability of the WB-effect for the description of gas-to-solid shifts of shape resonances. Finally, two minor bands cen- tered at $205.2 \mathrm{eV}$ and $209.8 \mathrm{eV}$ in the gas phase and 205.3 and $209.5 \mathrm{eV}$ in clusters are separated from the experimental spectra shown in Fig. 3. Gaussian distributions are used to model these bands. Their widths are found to be $\approx 7.0$ $\mathrm{eV}$ and $\approx 2.7 \mathrm{eV}$ in the molecular spectrum and $\approx 5.4 \mathrm{eV}$ and $\approx 1.7 \mathrm{eV}$ in clusters. In isolated $\mathrm{SF}_{6}$, these bands are expected to be due to double excitations. There, valence and Rydbergexcitations $\mathrm{Val}_{i} \rightarrow \mathrm{Val}_{j}$ and $\mathrm{Val}_{i} \rightarrow \mathrm{Ryd}$, may accompany the $\mathrm{S} 2 p$-to-valence transitions. ${ }^{15,37}$ The narrowing of these bands in clusters could be assigned to the suppression of $\mathrm{Val}_{i}$ $\rightarrow$ Ryd excitations, since it is well-known for molecular clusters that Rydberg transitions are strongly affected by interactions with the nearest neighbor shell. ${ }^{38}$ The spin-orbit components of these bands are not separated and their shapes are not examined here because of their location beyond the $\Omega_{4 e_{g}}$-interval.

\section{CONCLUSIONS}

Close resemblance and distinct difference in SDOS for the $\mathrm{S} 2 p$-to-valence transitions in free $\mathrm{SF}_{6}$ and $\mathrm{SF}_{6}$-clusters are revealed. No spectral gas-to-cluster shift is observed for the $\mathrm{S} 2 p \rightarrow 6 \mathrm{a}_{1 \mathrm{~g}}$-transitions and the band shape remains unchanged upon cluster formation. Significant changes are observed for $\mathrm{S} 2 p_{3 / 2,1 / 2} \rightarrow 2 \mathrm{t}_{2 \mathrm{~g}}$ - and $4 \mathrm{e}_{\mathrm{g}}$-transitions which are embedded in the $\mathrm{S} 2 p$-ionization continuum. Specifically, it is observed that the spectral redshift increases with excitation energy. In addition to these spectral redshifts, changes in band shape are observed. A de-convolution involving asymmetric and symmetric Lorentzians as well as Gaussian line profiles is applied in order to analyze these resonance bands. This allows us to extract the spin-orbit components of the narrow $2 \mathrm{t}_{2 \mathrm{~g}}$ and broad $4 \mathrm{e}_{\mathrm{g}}$-shape resonances and examine their spectroscopic characteristics. The dominating role of the asymmetric Lorentzian $L_{\Gamma}^{\alpha}(k)$ in shape of the $\mathrm{S} 2 p_{1 / 2,3 / 2} \rightarrow 2 \mathrm{t}_{2 \mathrm{~g}}-$ and $4 \mathrm{e}_{\mathrm{g}}-$ bands underlines their assignment to shape resonances.

Comparing the spin-orbit components of the $\mathrm{S} 2 p_{1 / 2,3 / 2}$ $\rightarrow 2 \mathrm{t}_{2 \mathrm{~g}}$ - and $4 \mathrm{e}_{\mathrm{g}}$-bands yields that (i) the gas-to-cluster redshift increases from $\Delta E_{0}=40 \pm 5 \mathrm{meV}$ up to $\Delta E_{0}=150$ $\pm 5 \mathrm{meV}$, (ii) the gas-to-cluster change in the Lorentzian width increases from $\Delta W_{\Gamma}^{2 p}=10 \mathrm{meV}$ up to $\Delta W_{\Gamma}^{2 p}$ $=60 \mathrm{meV}$, and (iii) the line asymmetry decreases in clusters for the both shape resonances. The mechanism of the gas-tocluster changes is assigned in clusters to the WB-effect on core-to-valence transitions which are embedded into the continua. This is due to the interference of the primary photoelectron wave with multiply scattered waves within the molecular cage and with waves singly scattered from neighboring molecules in clusters.

\section{ACKNOWLEDGMENTS}

Financial support by Deutsche Forschungsgemeinschaft (DFG) (RU 420/8-1 and Graduiertenkolleg 1582, TP A1) is gratefully acknowledged. Generous support by the GermanRussian Interdisciplinary Science Center (G-RISC) funded by DAAD and German Foreign Office (Grant Nos. P-2010a-7, 
P-2010b-7, P-2011a-3, P-2012b-21) as well as the LeonhardEuler-Program of DAAD is gratefully acknowledged. This work was also supported by NIR Grant No. 11.15.890.2012.

${ }^{1} \mathrm{H}$. Haberland, Clusters of Atoms and Molecules I (Springer, Berlin, 1995).

${ }^{2} \mathrm{H}$. Haberland, Clusters of Atoms and Molecules II (Springer, Berlin, 1996).

${ }^{3}$ E. Rühl, C. Heinzel, H. Baumgärtel, and A. P. Hitchcock, Chem. Phys. 169, 243 (1993).

${ }^{4}$ J. Wörmer, R. Karnbach, M. Joppien, and T. Möller, J. Chem. Phys. 104, 8269 (1996).

${ }^{5}$ O. Björneholm, F. Federmann, F. Fössing, and T. Möller, Phys. Rev. Lett. 74, 3017 (1995).

${ }^{6}$ R. Flesch, E. Serdaroglu, F. Blobner, P. Feulner, X. O. Brykalova, A. A. Pavlychev, N. Kosugi, and E. Rühl, Phys. Chem. Chem. Phys. 14, 9397 (2012).

${ }^{7}$ R. Flesch, A. A. Pavlychev, J. J. Neville, J. Blumberg, M. Kuhlmann, W. Tappe, F. Senf, O. Schwarzkopf, A. P. Hitchcock, and E. Rühl, Phys. Rev. Lett. 86, 3767 (2001).

${ }^{8}$ A. A. Pavlychev, X. O. Brykalova, R. Flesch, and E. Rühl, Phys. Chem. Chem. Phys. 8, 1914 (2006).

${ }^{9}$ A. A. Pavlychev, X. O. Brykalova, D. A. Mistrov, R. Flesch, and E. Rühl, J. Electron Spectrosc. Relat. Phenom. 166-167, 45 (2008).

${ }^{10}$ E. Rühl, Int. J. Mass Spectrom. 229, 117 (2003)

${ }^{11}$ I. L. Bradeanu, N. Kosugi, R. Flesch, and E. Rühl, J. Phys. Chem. A 112, 9192 (2008).

12 J. L. Dehmer, J. Chem. Phys. 56, 4496 (1972).

${ }^{13}$ D. Blechschmidt, R. Haensel, E. E. Koch, U. Nielsen, and T. Sagawa, Chem. Phys. Lett. 14, 33 (1972).

${ }^{14}$ J. L. Dehmer, J. Siegel, and D. Dill, J. Chem. Phys. 69, 5205 (1978).

${ }^{15}$ E. Hudson, D. A. Shirley, M. Domke, G. Remmers, A. Puschmann, T. Mandel, C. Xue, and G. Kaindl, Phys. Rev. A 47, 361 (1993).

${ }^{16}$ F. A. Gianturco, U. Lamanna, and C. Guidotti, J. Chem. Phys. 57, 840 (1972).

${ }^{17}$ M. N. Piancastelli, J. Electron Spectrosc. Relat. Phenom. 100, 167 (1999).

${ }^{18}$ I. Kojima and M. Kurahashi, J. Electron Spectrosc. Relat. Phenom. 42, 177 (1987).

${ }^{19}$ A. Losev, Surf. Interface Anal. 14, 845 (1989).

${ }^{20}$ O. Echt, D. Kreisle, E. Recknagel, J. J. Saenz, R. Casero, and J. M. Soler, Phys. Rev. A 38, 3236 (1988).

${ }^{21}$ R. N. S. Sodhi and C. E. Brion, J. Electron Spectrosc. Relat. Phenom. 34, 363 (1984).
${ }^{22}$ V. I. Nefedov, Zh. Strukt. Khim. 11, 299 (1970).

${ }^{23}$ P. Decleva, G. Franzoni, A. Kivimäki, J. Alvarez Ruiz, and S. Svensson, J. Phys. B 42, 055102 (2009).

${ }^{24}$ S. W. J. Scully, R. A. Mackie, R. Browning, K. F. Dunn, and C. J. Latimer, J. Phys. B 35, 2703 (2002).

${ }^{25}$ A. A. Pavlychev, J. Phys. B 32, 2077 (1999).

${ }^{26}$ A. De Fanis, N. Saito, A. A. Pavlychev, D. Y. Ladonin, M. Machida, K. Kubozuka, I. Koyano, K. Okada, K. Ikejiri, A. Cassimi et al., Phys. Rev. Lett. 89, 023006 (2002).

${ }^{27}$ T. Jahnke, J. Titze, L. Foucar, R. Wallauer, T. Osipov, E. P. Benis, O. Jagutzki, W. Arnold, A. Czasch, A. Staudte et al., J. Electron Spectrosc. Relat. Phenom. 183, 48 (2011).

${ }^{28}$ M. Kitajima, M. Hoshino, M. Okamoto, T. Suzuki, H. Tanaka, Y. Shimuzu, Y. Muramatsu, H. Chiba, K. Ueda, T. Hayaishi et al., Phys. Rev. A 63, 050703 (2001).

${ }^{29}$ M. N. Piancastelli, D. W. Lindle, T. A. Ferrett, and D. A. Shirley, J. Chem. Phys. 86, 2765 (1987).

${ }^{30}$ T. A. Ferrett, D. W. Lindle, P. A. Heimann, M. N. Piancastelli, P. H. Kobrin, H. G. Kerkhoff, U. Becker, W. D. Brewer, and D. A. Shirley, J. Chem. Phys. 89, 4726 (1988).

${ }^{31}$ J. L. Dehmer, D. Dill, and A. C. Parr, in Photophysics and Photochemistry in the Vacuum Ultraviolet, edited by S. P. McGlynn, G. L. Findley, and R. H. Huebner (Reidel, Dordrecht, 1985), p. 341.

${ }^{32}$ D. A. Mistrov, A. De Fanis, M. Kitajima, M. Hoshino, H. Shindo, T. Tanaka, Y. Tamenori, H. Tanaka, A. A. Pavlychev, and K. Ueda, Phys. Rev. A 68, 022508 (2003).

${ }^{33}$ Y. I. Kan, X. O. Brykalova, Y. S. Krivosenko, and A. A. Pavlychev, in Proceedings of the International Workshop on High-resolution Spectroscopy of Isolated Species: The Present and Future (HRSIS2012), St. Aubin, France, 14-15 September 2012.

${ }^{34}$ C. Leibold, H. Oberhammer, T. D. Thomas, L. J. Saethre, R. Winter, and G. L. Gard, Inorg. Chem. 43, 3942 (2004).

${ }^{35}$ A. S. Vinogradov, A. Y. Dukhnyakov, T. M. Zimkina, V. M. Ipatov, I. V. Karunina, D. E. Onopko, A. A. Pavlychev, S. A. Titov, and E. O. Filatova, Fiz. Tverd. Tela 22, 2602 (1980).

${ }^{36}$ A. S. Vinogradov, S. I. Fedoseenko, S. A. Krasnikov, A. B. Preobrajenski, V. N. Sivkov, D. V. Vyalikh, S. L. Molodtsov, V. K. Adamchuk, C. Laubschat, and G. Kaindl, Phys. Rev. B 71, 045127 (2005).

${ }^{37}$ A. Kivimäki, J. A. Ruiz, P. Erman, P. Hatherly, E. M. Garcia, E. Rachlew, J. R. I. Riu, and M. Stankiewicz, J. Phys. B 36, 781 (2003).

${ }^{38}$ E. Rühl, B. Brutschy, and H. Baumgärtel, Chem. Phys. Lett. 157, 379 (1989). 